\title{
Research of Rotor Unmanned Aerial Vehicle (UAV) Production and GIS Integration Based on 3D Panorama
}

\author{
Jingsi Li ${ }^{1,2,3,4, *}$, Jian Wang 1,2,3,4, Chenxi Yang 1,2,3,4 \\ ${ }^{1}$ Institute of Land Engineering and Technology, Shaanxi Provincial Land Engineering Construction Group Co., Ltd., Xi'an Shaanxi \\ ${ }^{2}$ Shaanxi Provincial Land Engineering Construction Group Co., Ltd., Xi'an Shaanxi \\ ${ }^{3}$ Key Laboratory of Degraded and Unused Land Consolidation Engineering, the Ministry of Nature and Resources, Xi' an Shaanxi \\ ${ }^{4}$ Shaanxi Provincial Land Consolidation Engineering Technology Research Center, Xi'an Shaanxi
}

\begin{abstract}
D panorama technology is a kind of virtual reality technology based on image creation, which is used real photos to show the scene to achieve the effect of immersive virtual roaming, and improve the interactivity between the network platform with the user. Panoramic images, UAV remote sensing and GIS technology is the application and development of new technology of surveying and mapping industry, but apply combination is rarely studied. In this paper, based on rotor unmanned aerial vehicle (UAV) fixed-point hover took photos of different angles using PTGui software to create panorama images stitching, optimization, software reuse Pano2VR making 3D roaming figure, and compared with Supermap GIS integration. Based on a downtown building community will be released on GIS 3D roaming figure, you can see, after the integration of $3 \mathrm{~d}$ panorama with two-dimensional GIS geospatial information, also can see the area the real scene. The surveying and mapping, tourism and other industries to provide a new technical method.
\end{abstract}

keywords: Panoramic image, 3D roaming figure, Rotor unmanned aerial vehicle, GIS integration

\section{Introduction}

Geographic Information System (GIS) is one of the fastest growing and most widely used technologies in various industries in recent years. The traditional thematic maps and vector maps generated based on GIS are twodimensional images, which can only obtain geospatial information and can not reflect objective things. The emergence of 3D modeling makes up for this deficiency, but the 3D modeling process is cumbersome and timeconsuming, which is not conducive to network transmission (LI,2013). The three-dimensional roaming map constructed by panorama can reflect the real scene, and can view things at any position and angle through mouse or keyboard (FENG,2013). Combining the threedimensional roaming map with the electronic map database, the combination of GIS and virtual scene is realized through web embedded publishing (DENG,2014). In the past, the panorama was taken by fixing the digital camera or vehicle camera with a tripod, and the shooting range was small. The rapid development of UAV widens the way for panoramic shooting. The UAV has a series of advantages such as low cost, simple operation, fast image acquisition speed and high ground resolution. The multi rotor UAV is equipped with a high-definition SLR camera, hovering in the air at a fixed point to shoot multiple pictures at 360 degrees horizontally and nearly 120 degrees vertically, which is used to produce an all-round real map(ZHOU,2016).

Taking an urban area as the shooting base, this paper uses inspire1 Pro multi rotor UAV equipped with Zen X5 camera to hover at the height of $300 \mathrm{~m}$ in the center of the shooting area, takes 57 photos by adjusting the camera angle, loads the photos into PtGui software, generates panoramic images through alignment optimization splicing, and uses Photoshop software to make up the sky for the panoramic images, Load a complete panorama after processing into pano2 $\mathrm{vr}$ software to create 3D roaming map, and then integrate and publish it with GIS. The research aims to provide technical support for regional surveying and mapping, tourism publicity and other industries.

\section{Panoramic shooting of multi rotor uav}

UAV aerial photography technology takes the aircraft as the data acquisition platform and carries a high-resolution image acquisition system to obtain image data. It has the characteristics of low cost, high precision and flexible system acquisition(CHEN,2016). At present, UAVs can be divided into fixed wing and multi rotor. According to their advantages and disadvantages, it is concluded that fixed wing is suitable for patrol measurement of land and

\footnotetext{
* Corresponding author: 692302520@qq.com
} 
crops, while multi rotor is more suitable for fixed-point shooting.

Therefore, this shooting adopts "Wu" inspire1 Pro rotor type small UAV, with a maximum takeoff weight of $3500 \mathrm{~g}$, a maximum horizontal flight speed of $18 \mathrm{~m} / \mathrm{s}$ and a maximum flight altitude of $500 \mathrm{~m}$. The system is simple, convenient and flexible, and easy to popularize. The main parameters of the Zen X5 camera carried on the UAV are shown in Table 1.

Table.1 Camera Parameter Table

\begin{tabular}{cc}
\hline Essential Factor & Type \\
\hline Camera model & ZenmuseX5 \\
Focal length & $30 \mathrm{~mm}$ \\
Specifications & $4 / 3 \mathrm{IN} \mathrm{CMOS} \mathrm{sensor}$ \\
Pixel numbers & $1600^{*} 10^{4}$ \\
Maximum resolution & $4608 \times 3456$ \\
\hline
\end{tabular}

According to the specific characteristics of the shooting area, the flight altitude is set to $300 \mathrm{~m}$, the shooting interval angle of each circle is less than 30 degrees, the rotation angle of each circle is less than 30 degrees, and 10-16 images are taken in each circle, so as to ensure sufficient image overlap and facilitate subsequent image splicing and processing. The shooting time of panoramic image can be completed within 10 minutes, and clear and complete images can be obtained.

\section{Production of panorama and $3 d$ roaming map}

Panoramic images include cylindrical panorama, spherical panorama and cube panorama. The essence of the three models is to project the spliced and fused panoramic images onto different three-dimensional surfaces, and back project them according to the visual angle of the viewer, so as to obtain images of different visual fields(DONG,2015). According to the previous production experience, it is concluded that the spherical panorama is the closest to human vision, so this paper takes the production of spherical panorama as an example(GONG,2017).

\subsection{PTGui Image Mosaic}

PTGui is a widely used panorama making software, which is easy to operate and can also be used by non professionals. Firstly, the photos taken by the UAV are loaded into the software in order and aligned automatically(WU,2016). Due to the camera focusing problem and weather influence, some adjacent pictures cannot automatically identify the overlapping area, so it is necessary to add control points manually. Set the isometric spherical projection from the panorama editor and adjust the reference line to align the building seams(DIAO,2016). Then, the optimizer is used to repeatedly optimize the control points with large error until the accuracy requirements are met, as shown in Figure 1.

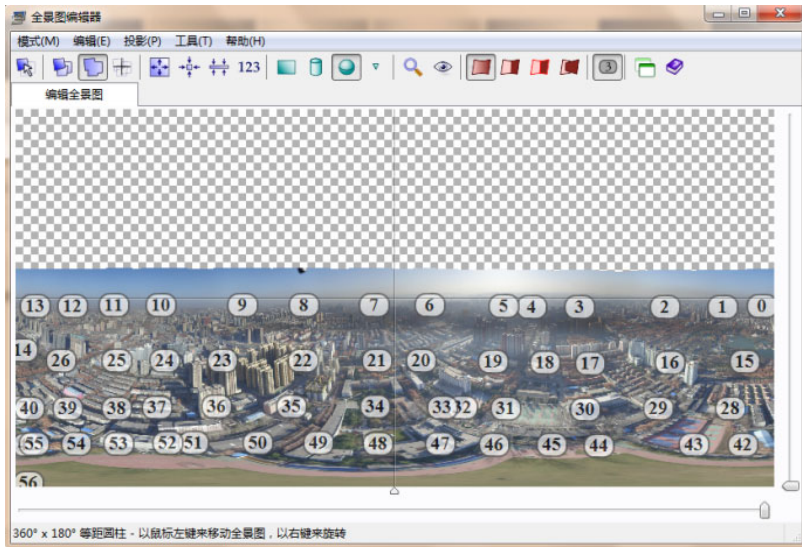

Figure 1. Image Splicing Adjust

\subsection{Image Patching in Photoshop}

When the UAV hovers in the air, the maximum elevation angle of the camera is 120 degrees, which can not illuminate the whole sky. Therefore, it is necessary to make up the sky on the ground, and then use Photoshop to splice and repair the sky picture and panorama, and color it(LIU,2012), as shown in Figure 2. Convert the panoramic picture and the sky picture into polar coordinates, integrate them into one picture, repair the seams, and use tools such as layer mask, gradient, imitation stamp and filling to achieve seamless combination.

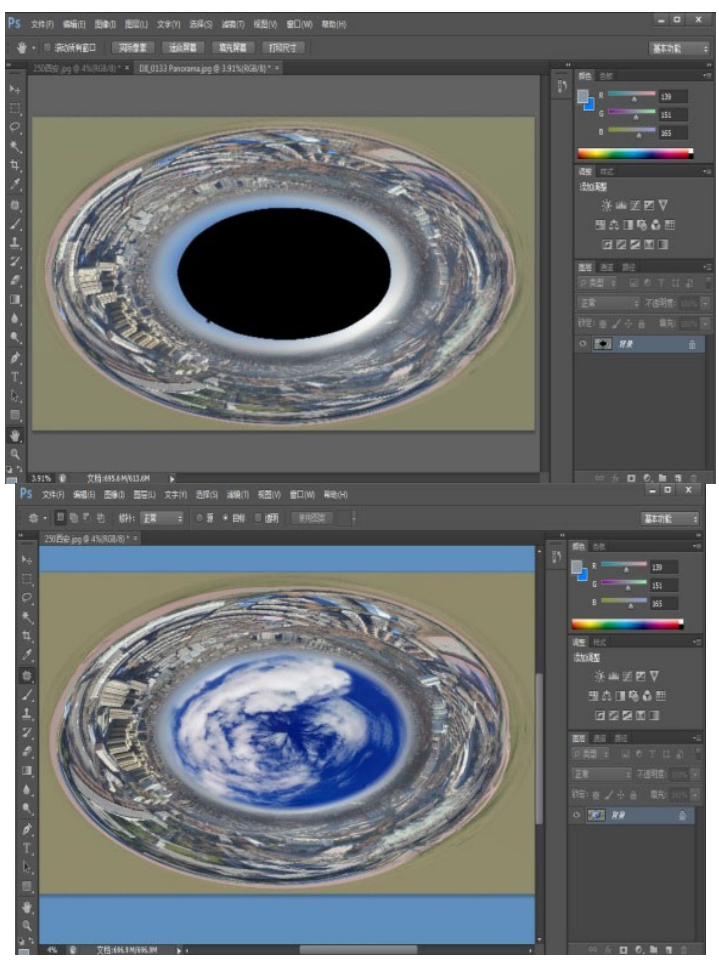

Figure 2. Under the Polar of Patch of the Sky

Then adjust the hue, saturation and contrast of the filled picture to make the picture clearer and the color more prominent. Finally, switch to the normal viewing angle and output the complete panoramic picture, as shown in Figure 3. 


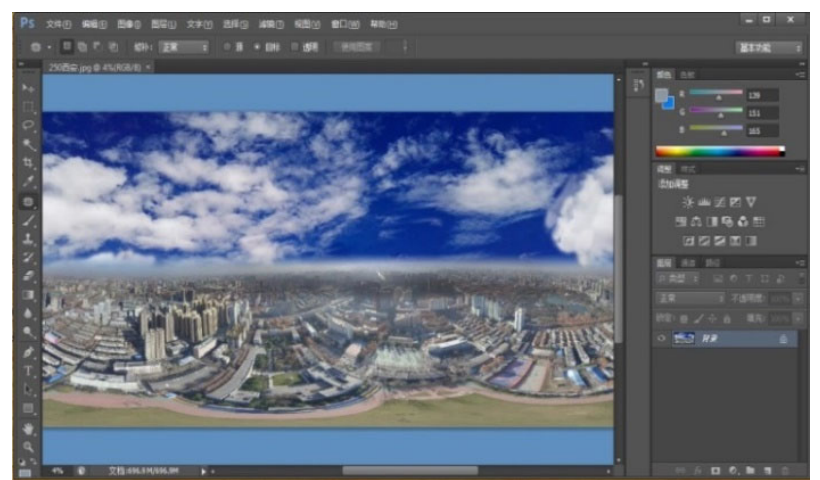

Figure 3. Complete Panorama

\subsection{Production of 3D Roaming Map}

Pano2vr is a panoramic image conversion application software based on flash animation technology. It can directly output animation files in Flash format by importing panoramic images in various formats and processing them(HUANG,2015).

Static panoramic pictures can not make people feel immersive, while 3D roaming pictures give people a new visual feeling and see wonderful images of different pictures from different angles(HUANG,2012). Load the panoramic image processed by PtGui and Photoshop software into pano $2 \mathrm{vr}$ software, set image parameters, change image quality and playback frame number, turn on other functions as required, and output in the form of flash and HTML. Both of them have low requirements for players and can be played on any computer, as shown in Figure 4(YE,2012). At the same time, when the scene is not single, you can connect the scene by adding links or hotspots. The things to be annotated and navigation paths in the scene can be represented by specific symbols by adding hotspots(LIANG,2013).

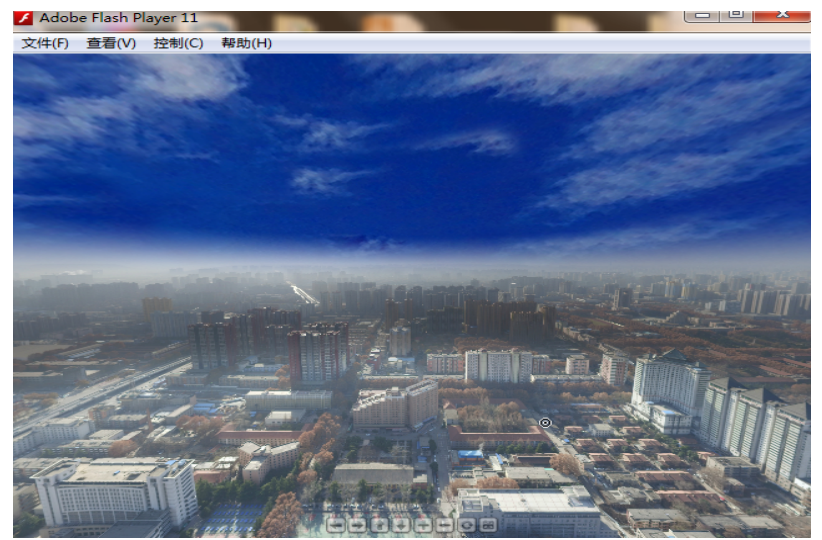

Figure 4. Flash 3D Roaming Figure

\section{Integration of $3 d$ panoramic roaming and gis}

The three-dimensional panoramic roaming map is published in the form of flash and HTML and embedded in various systems and web pages to show the real scene. However, you cannot view the geospatial and other attribute information of the image. GIS takes geospatial data as the core and realizes the interaction with users through professional interface. However, the image or map created based on GIS system itself is generally displayed in two-dimensional form. Even if the attribute data similar to DEM digital elevation map contains threedimensional information, it can not be displayed well in the interface, and the three-dimensional modeling of GIS is very cumbersome. Therefore, the emergence of $3 \mathrm{D}$ panorama and GIS integration technology makes up for this defect. The 3D panorama is integrated with GIS to give spatial coordinates on the panorama and establish a unified database(GUO,2013).

Based on the vector electronic map on SuperMap GIS, this paper embeds the $3 \mathrm{D}$ panoramic roaming map. The position coordinates of the three-dimensional panorama are corresponding to the electronic map and an icon is added. Users can click the icon to enter the 3D panorama to realize scene roaming, as shown in Figure 5.

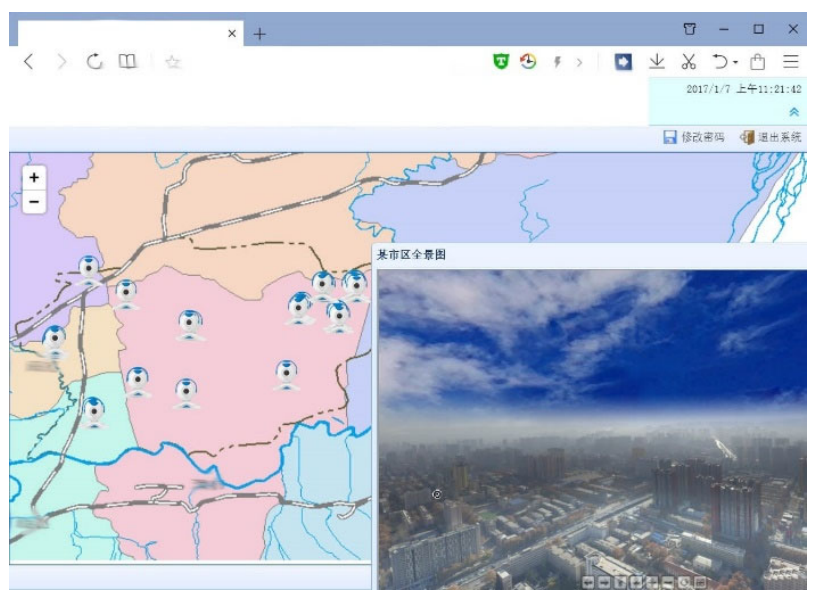

Figure 5. Panoramic Roaming and Gis Integration

\section{Conclusion}

The 3D panorama is processed by professional software PtGui and Photoshop to splice, fuse and repair the real image to generate a complete panorama. Then, the panoramic roaming map required by the user is generated through the parameter setting of pano2vr software. The rapid development of UAV provides a new and effective method for image data acquisition. Integrating 3D panoramic roaming map on GIS platform not only increases the geospatial information of 3D panorama, but also provides users with a better interactive platform. At the same time, the method discussed in this paper will be more widely used in surveying and mapping, tourism and other industries.

\section{References}

1. LI Shilei, DU Mingyi, LIU Yanwei, et al. The Research of the GIS Integration and Panorama Image[J]. Urban Geotechnical Investigation \& Surveying. 2013(4). 
2. FENG Jian, WU Lihua. Construction of the Tree-dimensional Panoramic Walk through Stem[J]. Computer \& Digital Engineering. 2013(1):115.

3. DENG Feng, WAN Yuan, LEI Yu, et al. Online Roaming and GIS Integration Research and Development Based on Three-dimentional Panoramic[J]. Acta Scientiarum Naturalium Universitatis Nankaiensis. 2014(4):47.

4. ZHOU Zhiyong,DING Yulan. Research of Panoramic Maps Based on Multi-rotor UAV[J]. Urban Geotechnical Investigation \& Surveying. 2016(2).

5. CHEN Wenlong, ZHANG Yu, YE Song, et al. Application of UAV Panoramic Technology to Site Monitoring[J]. Journal of Yangtze River Scientific Research Institute. 2016(11):33.

6. DONG Xiaoyong. The Application and Research of Three-dimensional Panorama Technology in Modern Agriculture Extension[D].Northwest A\&F University,2015.

7. GONG Qihui, WU Jianping, WANG Jiehua. The Research of the Production of 3D Real Scene and the GIS Integration Thereof Based on Panorama[J]. Geomatics \& Spatial Information Technology. 2012(6):35.

8. WU Xiaodong, WEN Peng, GUI Lin. Application of 360 Panoama Technology in the Hydro Power Engineering[J]. Geospatial Information.2016(5):14.

9. DIAO Zhigang, Liu Zhinong, Wang Ningning.Use PT-Gui Software Product Panorama[J]. Information science.2016(7).

10. LIU Lang. The Usage of Photoshop CS5 Panoramic Photo[J]. Science Mosaic. 2012(11).

11. HUANG Guozheng. Research on Interactive Campus Roaming System Based on Pano2VR[J].Software Engineer.2015.(18):1.

12. HUANG Xianbing. The Design and Implement of Online Roaming Landscape Based on Panoramic Technology [D].Shanghai: Shanghai Jiao Tong University,2012.

13. YE Pingping, LUO Hong. Manufacture Methods and Application of Three -dimensional Panoramagram Based on PTGui Pro、 Pano2VR[J]. Urban Geotechnical Investigation \& Surveying. 2012(4).

14. LIANG Lijing, LIAO Chaowei. Realization of $360^{\circ}$ Panoramic Roaming Animation Based on Static Images $[\mathrm{J}]$. Journal of Ping Xiang University.2013(30):6.

15. GUO Lin-gang, ZHOU Jie, ZHANG Bing, YANG Jing, et al. Application of GIS Integrated 3D Panorama in Environmental Emergency[J]. Environmental Science Survey. 2013(32). 